\title{
CHARACTERIZATION AND TUNING OF A MICROWAVE GUN CAVITY
}

\author{
W.K. Lau, B.J. Chan, L.H. Chang, C.W. Chen, H.Y. Chen, S.Y. Hsu, K.T. Hsu, J.Y. Hwang, Y.C. \\ Wang, T.T. Yang, NSRRC, Hsinchu, Taiwan
}

\begin{abstract}
A microwave electron gun system is being constructed to evaluate the feasibility of using such system as the electron source for the linac pre-injector in storage ring synchrotron radiation sources and for novel linac-based light source R\&D at NSRRC. A $2856 \mathrm{MHz}$ SSRL-type microwave gun cavity has been fabricated in house for this purpose. Properties of the cavity resonant modes have been characterized as a function of side-coupled cell plunger position. Special tuning plunger design for this cell allows re-adjustment of field ratio even after brazing. Field amplitude ratio of the cavity full cell to half cell has now been tuned to 2:1. That is the optimized ratio for low energy bunch compression by an alpha magnet.
\end{abstract}

\section{INTRODUCTION}

Thermionic cathode microwave gun system has reasonably high brightness as well as long cathode life time that can be used as a reliable electron source for the pre-injector in a $3^{\text {rd }}$ generation light source $[1,2]$. Microwave guns are also useful for novel light source $\mathrm{R} \& \mathrm{D}$ such as generation of intense coherent $\mathrm{THz}$ radiations, free electron lasers in the infra-red range and fs hard X-ray via Thomson scattering etc. [3, 4].

At NSRRC, an $2856 \mathrm{MHz}$ injector system is being constructed for evaluation purposes. Possible applications include TLS pre-injector upgrade, pre-injector for the proposed 3.3 GeV Taiwan Photon Source (TPS). This linac injector system has a SSRL microwave gun, an alpha magnet for bunch compression and two sections of S-band linacs for electron acceleration. Figure 1 depicted the microwave gun cavity that has been machined and brazed recently at NSRRC by the radio frequency group and is now under cathode test.

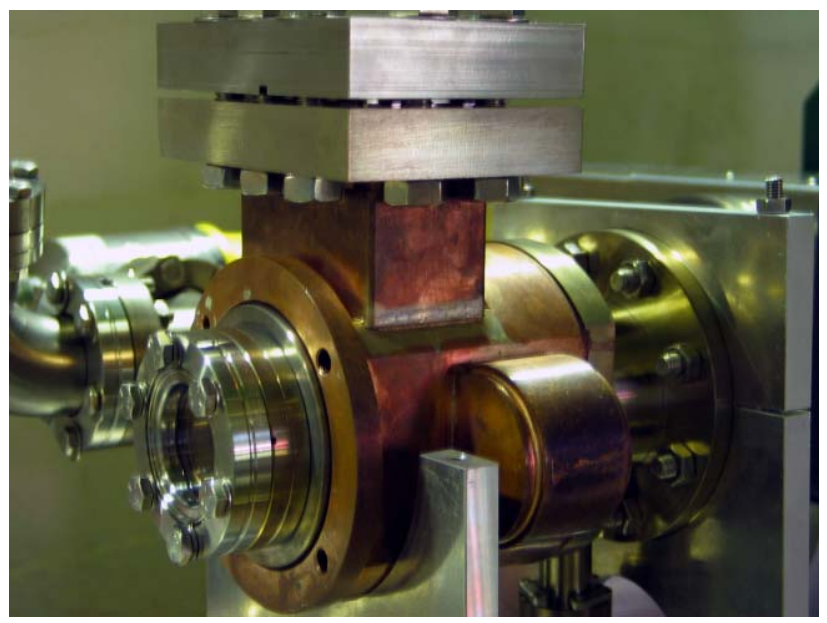

Figure 1: The $2856 \mathrm{MHz}$ microwave gun cavity fabricated in house at NSRRC.
Since the original SSRL microwave gun thermionic cathode (with nose cone) design has been improved for easy installation and commercialized by HeatWave Labs, Inc. $[5,6]$ as an integrated assembly (Figure 2), we purchase the cathode assembly from this company so that efforts to develop this cathode assembly has been minimized.

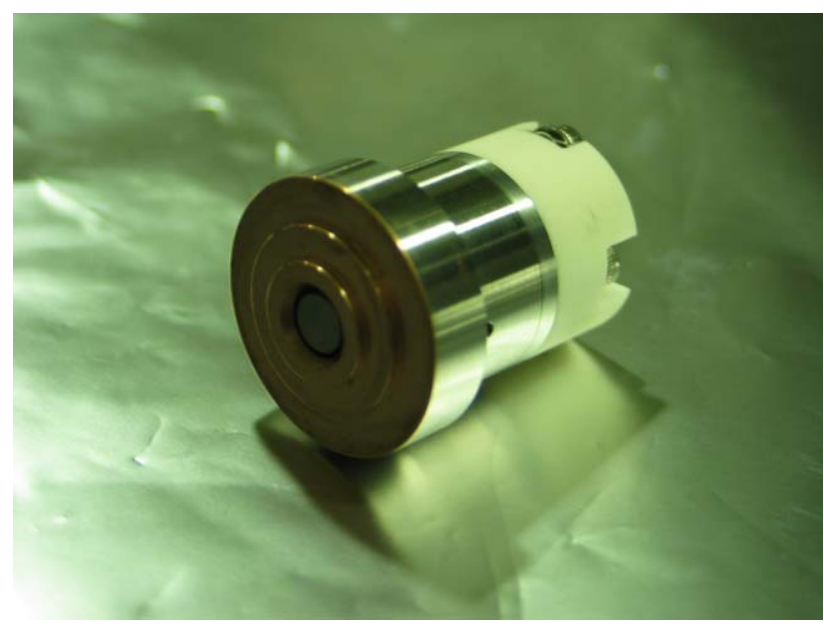

Figure 2: Thermionic cathode (with nose cone) assembly purchased from HeatWave Labs, Inc.

The cathode assembly will be installed at the end wall of the half-cell of the microwave gun cavity and this cell is coupled to the full cell through a side-coupled cell which is not on a path along the beam axis. Therefore, the gun cavity is a resonant structure with three eigenmodes. They are the 0 -mode, $\pi / 2$-mode and the $\pi$-mode. Klystron power will be fed into the cavity at the side wall of the full cell through an iris. Coupling of klystron power to the cavity modes are in general depend not only on iris size and the individual electromagnetic properties of each cell and, but also on the coupling between cells.

In this paper, we report the results of bench measurements we have done to characterize the properties of the cavity for each eigenmode such as resonant mode frequency, Q values, coupling coefficients as functions of the side-coupled cell plunger position which is related to coupling strength of full cell to half cell. The cavity field ratio is usually adjusted to a certain value for special application. For example, field ratio can be optimized for bunch compression. Adjustment of field ratio is achieved by tuning the side-coupled cell resonant frequency with tuner plunger and hence the coupling coefficients and Q values of all modes will be changed accordingly. A special plunger design allows re-adjustment of field ratio even after brazing of the microwave gun cavity so that the cavity can be re-used for a different field ratio setting. 


\section{CHARACTERIZATION OF THE MICROWAVE GUN CAVITY}

The microwave gun cavity has been brazed and leak checked before bench measurement. Throughout the measurement, a machined aluminium disc which has the dimensions that are identical to the cathode (with nose cone) has been put in place to block the hole for the real cathode. From the measured $S_{11}$ data of the waveguide input port, the frequencies, quality factors and coupling coefficients of all three modes are derived.

\section{Frequencies and Coupling Coefficients of the Cavity Eigenmodes}

The reference position "zero" is defined as the position where the tuning plunger is inserted all the way into the coupling cell. As the plunger is pulled out from the coupling cell away from the reference position, the plunger position is considered as positive. Circuit analysis of a three coupled LRC oscillators [7] predicted that there are three eigenmodes in the gun cavity. The $\pi$-mode has the highest resonant frequency and the 0-mode has the lowest resonant frequency for all cell coupling strengths (plunger positions). However, the $\pi / 2$-mode frequencies is less sensitive to the changes in plunger positions and this mode is considered to be less sensitive to cell frequency errors as well as other perturbations in the cells. Our observation (Figure 3) agrees with the prediction of the circuit analysis.

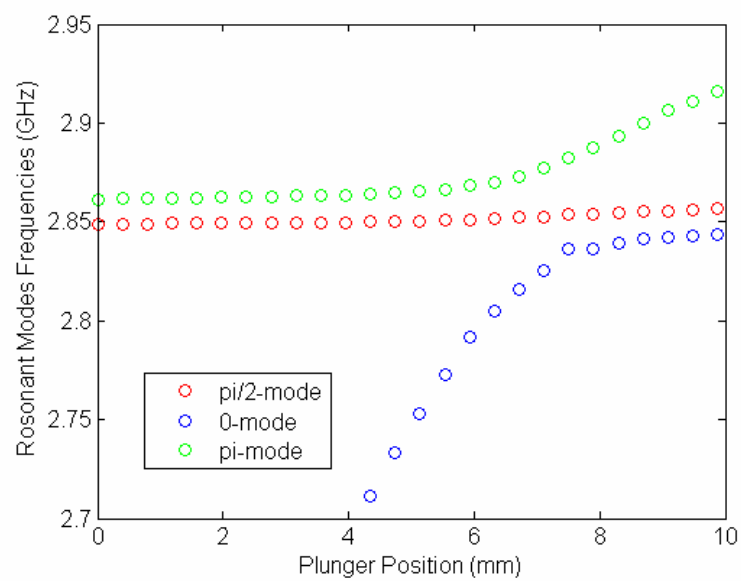

Figure 3: Dependence of eigenmode frequencies on sidecoupled cell tuning plunger positions.

When the plunger position is smaller than $\sim 5 \mathrm{~mm}$, the 0 mode and $\pi$-mode are very weakly coupled (under coupled) to the input waveguide. The $\pi / 2$-mode is over coupled to external circuit and the coupling ratio is about 8 (which is 4 for a un-brazed cavity). For plunger positions less than $\sim 5 \mathrm{~mm}$, basically only the $\pi / 2$-mode will be excited. Measured unloaded Q values are about 15,000 (Figure 5) and in good agreement with the ohmic $\mathrm{Q}$ as calculated by SUPERFISH for the full cell. Therefore, one can conclude that only the full cell is excited in this situation. As the plunger position is increased to values larger than $5 \mathrm{~mm}$, coupling to 0 -mode and $\pi / 2$-mode become stronger. Coupling to $\pi$-mode has no significant changes. It is believed that this coupling scheme is favourable to the $\pi / 2$-mode. However, as shown in figure 4 , measured coupling coefficients for all three resonant modes are functions of plunger positions. Therefore, coupling coefficient depend on both iris size and coupling strengths among cells.

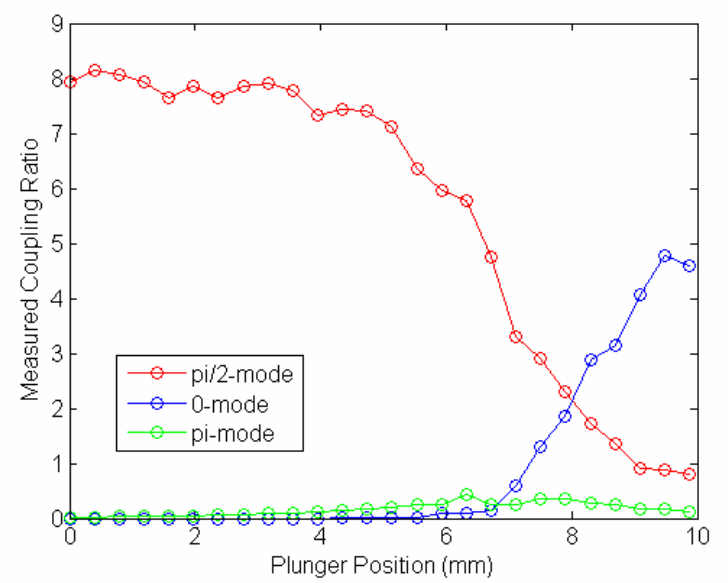

Figure 4: Measured coupling coefficients of the resonant modes versus plunger positions.

\section{De-Qing of $\pi / 2$-mode by Coupling Cell Tuning}

The coupling coefficient for the $\pi / 2$-mode decreases as plunger position increases because power loss in the microwave cavity increases as coupling to the sidecoupled cell and half cell become stronger. The measured unloaded Q is a decreasing function of plunger position justified this interpretation. We referred this effect as deQing of microwave gun cavity by side-coupled cell tuning. As coupling coefficient approaches one, the external microwave has maximum power transfer to the cavity and the value of unloaded $Q$ in this case is minimum. However, unloaded $\mathrm{Q}$ increases again as $\beta<1$ (under coupled). In practice, one needs consider this de-Qing effect if one wants to adjust the cavity to a certain field ratio but keeping the cold cavity overcoupled to minimize reflection when the cavity is loaded by the beam.

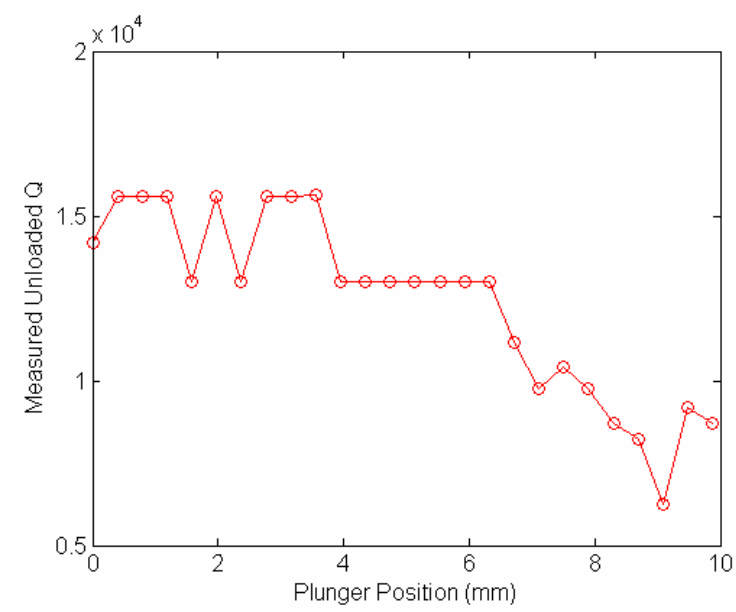

Figure 5: De-Qing of $\pi / 2$-mode by coupling cell tuning. 


\section{TUNING FOR OPTIMUM FIELD RATIO}

It has been investigated by S. Rimjaem et al. that when the field amplitude of the full cell approximately equals to two times that of the half cell, the electron beam has a linear energy chirp that is ideal for bunch compression with alpha magnet $[8,9]$. We therefore decided to set the field ratio as 2:1 for a first operation in near future. Field mapping has been done in term of bead-pull measurement, a $3 \mathrm{~mm}$ diameter copper bead is used as the perturbation along the cavity axis (we assumed there is no magnetic field along the cavity axis). The motion of this bead along the axis is driven by a PC controlled stepper motor. Since field amplitude is proportional to the square root of frequency shift due to the bead perturbation over the unperturbed resonant frequency, amplitude of the microwave gun has been mapped along the beam axis as the copper bead traverses the cavity mechanical axis. Figure 6 is the field map after cavity tuning for optimum field ratio. Unloaded $Q$ of the $\pi / 2$-mode under such cavity field ratio is about 9000 and coupling ratio of external circuit to the cavity is about $2 \sim 3$. We expect the cavity can still have a good matching to the waveguide under a beam loaded condition.

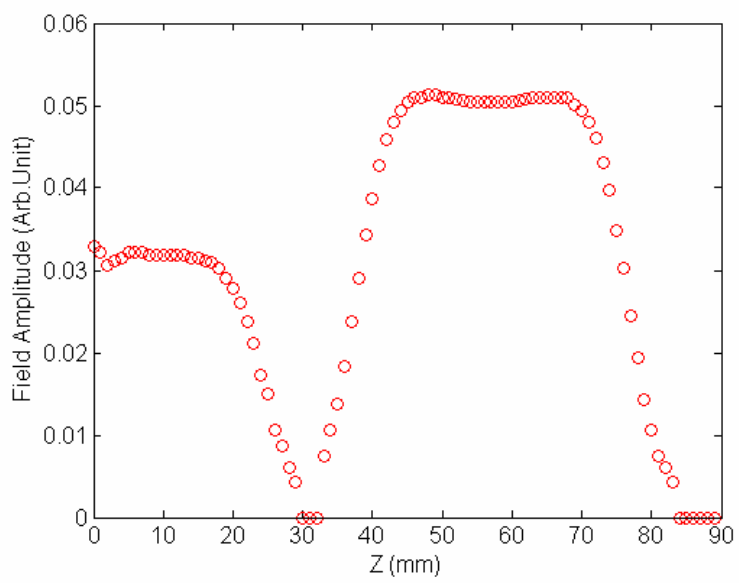

Figure 6: Measured electric field amplitudes (arbitrary unit) as a function longitudinal position. The position zero is the location of the thermionic cathode.

\section{SUMMARY}

A microwave gun cavity has been fabricated and vacuum tested. Properties of each of the three eigenmodes have been observed and characterized as a function of side-coupling cell plunger position.

Field ratio has been tuned to 2:1 for first operation of the microwave gun for generation of short electron bunches. A microwave gun system for a test injector is being installed at NSRRC, we expected to see a first beam from the gun by the end of this year. Themionic cathode has been installed and being tested in the following weeks. Full installation will be started in June.

The authors would like to thank Prof. Helmut Wiedemann not only for his enthusiasm to provide as much technical details as we requested about the microwave gun injector but also for his patient to explain to us the interesting beam dynamics in an rf gun and low energy beam compression using alpha magnet.

\section{REFERENCES}

[1] J.W. Lewellen et al., "Operation of the APS RF Gun" Proceedings of LINAC98', p.863-865.

[2] S. Park, "Thermionic RF Gun and Linac Pre-injector for SPEAR3" Proceedins of LINAC 2002, p.322-324.

[3] G.L. Carr et al.,"High Power Terahertz Radiation from Relativistic Electrons", Nature, Vol. 420, 2002.

[4] K. Nakajima et al., "A Short Pulse X-ray Generation via Thomson Scattering of a Ultrashort Laser Pulses by Relativistic Electron Beam" Proceedings of PAC97', p.760-762.

[5] Helmut Wiedemann, private discussions.

[6] http://www.cathode.com

[7] T.P. Wangler, "Principles of RF Linear Accelerators", John Wiley \& Sons, Inc., NY, 1998.

[8] Helmut Wiedemann, private discussions.

[9] S. Rimjaem et al,"Femtosecond Electron Bunches from an RF Gun", NIMA 533, 2004, p.258-269. 